\title{
La science et la technique dans la publicité
}

\section{Marie-Hélène Fries-Verdeil}

\section{(2) OpenEdition}

\section{Journals}

Édition électronique

URL : http://journals.openedition.org/asp/4378

DOI : $10.4000 /$ asp. 4378

ISSN : 2108-6354

Éditeur

Groupe d'étude et de recherche en anglais de spécialité

\section{Édition imprimée}

Date de publication : 1 mars 1993

Pagination : 301-322

ISSN : 1246-8185

\section{Référence électronique}

Marie-Hélène Fries-Verdeil, «La science et la technique dans la publicité », ASp [En ligne], 1 | 1993, mis en ligne le 01 mai 2014, consulté le 02 mai 2019. URL : http://journals.openedition.org/asp/4378 ; DOI : 10.4000/asp.4378

Ce document a été généré automatiquement le 2 mai 2019.

Tous droits réservés 


\title{
La science et la technique dans la publicité
}

\author{
Marie-Hélène Fries-Verdeil
}

1 «Science et technique dans la publicité » est un titre qui englobe à la fois les publicités ayant un objet technique ou scientifique, et celles qui utilisent la science ou la technique comme argument publicitaire.

2 Cette étude constitue un compte-rendu d'expérience pédagogique faite auprès d'étudiants biologistes et pharmaciens de l'université Joseph Fourier à Grenoble. Le but consistait à utiliser la publicité, soit pour réactiver un contenu grammatical ou lexical, soit pour lancer ou approfondir une discussion. Les publicités choisies comme exemple sont tirées de divers magazines scientifiques britanniques ${ }^{1}$.

\section{La publicité à caractère scientifique ou technique comme support de réutilisation de structures grammaticales}

3 Il s'agit à ce stade de permettre de retravailler des fonctions déjà acquises par les étudiants dans leur manuel Minimum Competence In Scientific English (1991), en utilisant comme support des images au lieu de mots. Je développerai la comparaison, la causalité, et la modalité.

4 Pour la comparaison, prenons par exemple la publicité conçue pour l'entreprise de télécommunications Alcatel sous forme de quatre planisphères de plus en plus petits (cf. annexe 1). En montrant seulement l'image aux étudiants, et en posant la question «What's happening to the world?", on obtient la réponse: "The world is getting smaller ». On peut alors chercher ensemble les causes de cette impression de rétrécissement, et on obtient des phrases telles que :

Thanks to phones, the fax and electronic mail, we can get through to people all around the world much faster than we used to be able to. Computer modems make business to business communications more convenient. Plane trips make journeys 
shorter. Satellite television channels broadcast information and ideas to a wider audience. World fashion in buildings, clothes and music makes people feel nearer home, even in a foreign country.

5 Pour illustrer le mécanisme de causalité, les exemples retenus ont été choisis parmi les publicités de William Heath Robinson (1979), un dessinateur anglais dont les œuvres révèlent à la fois une connaissance en profondeur des industries pour lesquelles il travaillait (il faisait consciencieusement nombre de visites d'usines avant de se mettre à l'ouvrage), et un humour typiquement britannique.

6 L'objectif visé ici est la réutilisation de structures verbales présentées dans le chapitre 10 de Minimum Competences: Means and Process: to enable, allow, permit, make possible, let.

7 Les étudiants étaient regroupés par deux, et chaque paire disposait d'un quart d'heure pour décrire une affiche de W.H. Robinson, en utilisant, entre autres, les verbes ci-dessus, avant de venir présenter oralement le résultat de leur travail au rétro-projecteur. Voici quelques-unes de ces descriptions : (cf. annexes 2 et 3 )

Smith's sectric clock

This technical process allows people to be always on time. Thanks to this system, everybody can go to work at the same time. Smith's system makes it possible for the clocks to be accurate. The Greenwich meridian has been chosen as the origin of the time zones, thus enabling us to travel all over the world, knowing the time of every country.

In the filtering vaults at Kilmarnock

This drawing shows a strange method to distillate Johnnie Walker whisky. The first man is opening a tap, so as to let the liquid drip down. Each drop has to go through nets, sieves, funnels, sponges, a scarf, knickers, and so on. At the end of this journey, the drop can be recovered thanks to a spoon, which makes it possible for another man to carry it up a ladder and into a barrel. We are thus enabled to drink very good Johnnie Walker whisky thanks to all this hard work.

8 Pour ce qui est de la modalité, les images se révèlent souvent particulièrement utiles pour faire exprimer des degrés de probabilité. Il suffit de trouver une publicité un peu mystérieuse. Prenons par exemple le cas de la firme métallurgique Davis Standard, qui avait choisi de faire figurer, sur la première moitié d'une double page, une paire de chaussures en gros plan, suivie de l'accroche suivante: "When it's hard to be in your shoes ... » (cf. annexe 4). Le groupe peut alors explorer toute une palette de sens possibles, en utilisant les auxiliaires modaux : may, might, could... En réponse à la question : « What kind of business is this advert for? ", j'ai obtenu les hypothèses suivantes :

"it might be for a temporary work agency (it's hard to be in your shoes... because you lack the skilled manpower)"

ou bien

it may advertise for an express delivery service (it's hard to be in your shoes... because you cannot get the supplies you need in time.)

ou encore :

It could inform about new computers or software packages (it's hard to be in your shoes... because your computers and/or programs are out of date now, so your firm is getting less and less efficient every day.

Ces trois exemples ont donc permis de voir comment les publicités permettent la réactualisation de structures grammaticales de façon dirigée, et pourtant relativement spontanée, sans que les étudiants aient l'impression d'être acculés dans le carcan d'un exercice où l'on attend d'eux une réponse unique, puisque la multiplicité de sens de l'image rend possible un large éventail de réponses différentes. 


\section{L'acquisition de vocabulaire à travers les publicités à caractère scientifique}

10 Enseigner ou réactiver des éléments lexicaux dans ce contexte revient souvent à jouer sur le rapport entre texte et image publicitaire, qu'il se manifeste sous forme de complémentarité, de redondance, ou de décalage. La redondance est souvent le fait des publicités purement informatives, proches des notices techniques, qui se contentent de montrer un objet, de le nommer, de l'expliquer. Dans ce cas, le travail consiste essentiellement à établir des correspondances terme à terme entre éléments lexicaux et éléments icôniques (cf. annexe 5).

11 Lorsque le binôme image-texte publicitaire engendre une contradiction ou une incongruité, le décalage provient le plus souvent de la juxtaposition de deux univers différents. Prenons l'exemple des auvents Somfimatic (cf. annexe 6).

Alors que le texte publicitaire fournit une description technique précise desdits auvents, l'illustration humoristique qui l'accompagne rappelle au souvenir du lecteur l'histoire des trois petits cochons, et le monde de l'enfance. Le slogan: "Don't leave home without it " joue un rôle unificateur sur le ton de la parodie. «It » renvoie certes anaphoriquement à un auvent Somfymatic, mais évoque aussi une protection contre le souffle destructeur du grand méchant loup, et le slogan constitue lui-même un clin d'œil comique à la campagne publicitaire bien connue d'American Express.

13 Ce décalage sémantique correspond à des niveaux de langues différents, auxquels on peut sensibiliser les étudiants en leur demandant de transformer la publicité écrite qu'ils ont devant les yeux en annonce radiodiffusée, comme le fait par exemple notre collègue Janet Atlan à Nancy. Voici en illustration le texte de l'annonce radio conçue pour les auvents Somfymatic par une étudiante en deuxième année de biologie :

Once upon a time there were three little pigs. They liked awnings a lot, because they were fond of having a shaded area, a protection against the summer heat. But the big bad wolf was always coming to destroy the awnings. And he huffed and he puffed and he blew the awnings down. But now, this pathetic situation is over for the three little pigs: they have discovered the Somfymatic II awnings. These awnings are so technically sophisticated that they automatically retract when the big bad wolf tries to blow them down!

Le travail a consisté ici à réécrire toute l'annonce dans le niveau de langue correspondant à celui de l'image. Mais, au-delà de l'aspect retranscription, l'intérêt de cet exemple est de montrer que le décalage entre texte et image publicitaire peut constituer une porte ouverte sur la créativité, l'invention et le jeu.

Néanmoins, c'est quand même la complémentarité entre texte et image qui offre l'éventail de possibilités le plus étendu pour une exploitation lexicale des publicités, parce que c'est le cas de figure où l'on peut le mieux moduler le niveau de spécialisation du vocabulaire en fonction du groupe d'étudiants et de ses besoins. Prenons par exemple la publicité de Bayer expliquant leur démarche en recherche et développement, pour la conception de nouveaux médicaments (cf. annexe 7). Cette publicité a été introduite dans le cours de pharmacie de troisième année de Grenoble par Lorraine Grison, pour un public étudiant fort au fait des effets secondaires de beaucoup de traitements, mais qui n'avait plus eu de cours d'anglais depuis le baccalauréat. Pour un groupe faible, la description de l'image était prétexte à une révision de vocabulaire de base (doctor, patient, 
ill, illness, disease, medecine, drug, etc.). Pour des étudiants plus avancés, on pouvait au contraire insister sur le texte publicitaire.

Lorsqu'ils ont découvert, à partir de quelques questions simples (Who is the advert aimed at? What kind of firm does Bayer appear as?) que la cible visée était le grand public, et que Bayer cherchait à vulgariser les principes déontologiques qui président à l'élaboration de leurs nouveaux médicaments, ils ont pu facilement recoder le message en termes techniques :

to cause cell proliferation : to be carcinogenic

to damage the genetic make up: to be teratogenic, etc.

Les publicités peuvent donc fournir une palette variée de moyens d'élucidation du vocabulaire. Mais, le plus souvent, ces exercices lexicaux ne sont pas une fin en soi, et constituent seulement un premier pas avant de lancer une discussion.

\section{La publicité à caractère scientifique comme outil de communication}

Les textes publicitaires offrent rarement un point de vue assez exhaustif pour faire le tour d'un problème donné, mais leur côté partiel, voire partial, se double le plus souvent d'une pointe incisive qui « accroche » l'attention et provoque le débat.

Lorsque la discussion suit une information (par exemple après un exercice de compréhension de la presse écrite ou un exposé), la seule vue de la publicité constitue en général un choc déclencheur suffisant, quitte à insister au début sur un ou deux points significatifs. La campagne publicitaire lancée à l'automne dernier par British Nuclear Fuels est à cet égard très intéressante.

Pour la double page sur l'effet de serre (cf. annexe 8), on peut commencer par la question : "One of the words here has a double meaning. Which one? ", analyser le jeu de mots sur "plant»(flower/factory), et montrer comment l'image rend le double sens plus explicite encore en juxtaposant dans la serre usines et semis. L'insolite dû à l'introduction d'une maquette de zone industrielle dans le jardin d'une maison de banlieue britannique typique permet de comprendre que cette cage de verre est une métaphore pour la planète Terre toute entière, soumise à l'effet de serre. Ce qui conduit tout naturellement à un débat sur l'environnement.

Dans la publicité utilisant les pluies acides (cf. annexe 9), j'avais choisi comme question tremplin: "There is a contradiction between the picture and the text, which one?». La contradiction naît ici bien sûr du choc entre le bon sens populaire de l'accroche (une goutte de pluie n'a jamais fait fondre personne), et le caractère incongru de l'image (ces citrons, ces bouteilles de vinaigre et ces batteries de voitures qui dégringolent du ciel, comme une actualisation et une métamorphose perfide de l'expression : «it's raining cats and dogs »). La contradiction vise bien sûr à expliquer en termes simples (et peut-être simplificateurs) les dangers dus aux pluies acides. De là à conclure que des centrales nucléaires à risque, mais non polluantes, sont après tout préférables à des centrales thermiques qui causent des pluies acides, il n'y a qu'un pas, et toute la publicité vise à nous le faire franchir, ce qui donne en tout cas ample matière à discussion.

Dans chacun des cas pré-cités, le point de départ était, grâce aux images, assez simple et assez polémique pour permettre à chaque étudiant, quel que soit son niveau, d'exprimer son point de vue. Ce qui n'exclut pas, bien sûr, de pouvoir ensuite insérer dans le débat 
des points plus complexes déjà vus dans l'exposé ou l'exercice de compréhension écrite précédent.

Lorsqu'on veut lancer la discussion à partir du seul support publicitaire, une analyse plus fouillée s'impose, que l'établissement d'une grille de lecture permet de guider. Dans la grille proposée en annexe, les emprunts à la communication publicitaire ont été réduits à deux concepts clés : le public-cible (qui permet d'identifier les destinataires du message) et l'image de marque (qui renvoie au contraire à la source du message, c'est-à-dire à l'annonceur). Ces deux concepts font l'objet d'une explication dans la grille elle-même, de façon à être clairement compris par un public n'ayant pas de connaissances particulières dans le domaine de la publicité. Les autres cases de la grille font appel à des points de repère plus axés sur l'aspect scientifique ou technique.

L'intérêt d'une telle grille est d'abord d'ordre pratique : elle permet un renouvellement fréquent des documents utilisés, sans surcroît de travail, puisqu'elle peut s'appliquer à n'importe quelle publicité à caractère scientifique ou technique. Au-delà de l'aspect pratique, la grille fournit une "mémoire ", et permet la comparaison entre plusieurs publicités (complémentaires ou concurrentes par exemple.). Pour l'instant, cette grille a toujours été utilisée en deux temps : tout d'abord, chaque étudiant disposait d'un moment pour finir de la remplir, après avoir vu chaque publicité deux ou trois fois. Cette première étape vise d'abord bien sûr à assurer une compréhension orale globale, mais elle laisse aussi du temps à chaque étudiant pour commencer à réfléchir. Ce temps de travail individuel est ensuite suivi d'une correction collective et d'une discussion en groupe. Pour un groupe de niveau faible, cette discussion peut se construire comme un développement de la correction, en cherchant à faire réutiliser aux étudiants les fonctions grammaticales induites par les questions de la grille. (cf. annexe 10 pour cette possibilité, développée à la suite d'une suggestion de notre collègue Jean Sabiron de Poitiers). Un groupe plus implicite et explicite, bref, jongler avec du sens.

En conclusion, il me semble que l'a priori défavorable dont souffre la publicité à caractère scientifique, qui apparait souvent comme purement informative, hyperspécialisée, dénuée de tout humour, ou de tout intérêt pour l'humain est loin d'être toujours fondé. Elle est soumise, bien sûr, aux lois du marché qui gouvernent les espaces publicitaires, mais ces contraintes financières, qui privent la publicité des lettres de noblesse gagnées par les documentaires, ou les articles de vulgarisation, ont l'immense avantage de lui conférer un caractère limité, et concentré. Limites il y a, fixées par le jeu entre les montants des budgets publicitaires, et la quantité de pages de magasines ou de minutes de télévision qu'ils peuvent acheter. La concentration devient alors règle d'or de la publicité, ce qui en fait un outil de complément très précieux pour l'enseignement de l'anglais de spécialité. L'image, d'une part, fournit aux étudiants, même faibles en anglais, un tremplin pour parler, et discuter. D'autre part, elle suggère sans imposer, et permet de moduler l'apport de vocabulaire général, de mots de spécialités et de structures grammaticales, en fonction des besoins du groupe, et de ses motivations.

Enfin, et ce n'est pas là le moindre avantage de la publicité, le débat qu'elle suscite permet d'utiliser fructueusement la complémentarité entre celui qui sait sans pouvoir parler (l'étudiant faible en anglais, mais doué dans sa spécialité), et celui qui parle, sans toujours connaître à fond l'arrière-plan scientifique sur lequel il s'appuie (le professeur de langues). 


\section{BIBLIOGRAPHIE}

Peninou, Georges. 1972. Intelligence de la publicité, étude sémiotique. Paris : Robert Laffont.

Robinson, William Heath. 1979. The Gentle Art of Advertising. Londres : Duckworth.

Upjohn, Jonathan, Sue Blattes et Véronique Jans. 1991. Minimum Competence in Scientific English.

Grenoble : Presses Universitaires de Grenoble.

Les publicités reproduites en annexe sont tirées des magazines suivants : Alcatel ( $\left.\mathrm{n}^{\circ} 1\right)$, New

Scientist Oct. 1991 ; David-Standard ( $\left.\mathrm{n}^{\circ} 4\right)$; Metallurgical Plant and Technology International Sept 1989 ;

Urethanes Technologies Oct.-Nov. 1989 ; Industrial Fabric Products Review April 1990 ; Bayer ( $\left.{ }^{\circ} 7\right)$,

New Economist Dec. 1991 ; British Nuclear Fuels (nº 8 \& 9) ; New Scientist Nov. 1991.

\section{ANNEXES}

\section{Annexe. Compte-rendu des débats}

Les interventions qui ont suivi ont d'abord porté sur le fond du problème. Monique Mémet (IUT, Université de Paris XII), s'est interrogée sur le principe d'élaboration de la grille d'interprétation générale, qui doit beaucoup aux copy strategies (ces documents établis par les publicitaires pour délimiter les attentes des annonceurs) et s'est demandée quel pouvait être le bien-fondé de son utilisation avec des étudiants non spécialistes de publicité. Pour Elizabeth Jolivet (Université d'Orléans), la question était plutôt de savoir dans quelle mesure il était possible de travailler avec des publicités sans enseigner préalablement l'analyse d'images. Jean Sabiron (Université de Poitiers) a insisté sur l'importance de définir des objectifs fonctionnels (de quels mots, de quelles structures grammaticales les étudiants ont-ils besoin pour répondre aux questions de la grille ?), et suggéré d'établir des correspondances entre les cases de la grille et les fonctions grammaticales définies dans Minimum Competence (op. cit.). Au-delà du questionnement sur la grille de lecture (qui a été pris en compte dans la rédaction finale de la communication), ces trois interventions posaient au fond le problème de savoir avec quels outils enseigner à lire, à décoder des publicités, et reprenaient comme en écho la question centrale que se posait Georges Péninou (1972:14) : «Comment le publicitaire fait-il advenir le sens aux choses?».

Il est difficile de prétendre répondre exhaustivement en quelques lignes, mais un élément de réponse très intéressant me semble avoir été donné par l'intervention d'Hubert Greven (Université de Savoie, président de séance), qui nous a rappelé l'importance essentielle d'une analyse paradigmatique dans les publicités, puisque celles-ci jouent souvent bien plus sur le non-vu et le non-dit que sur le vu et le dit. Or, cette analyse de ce qui aurait pu être dans l'icône et dans le texte, et qui pourtant n'y figure pas, fait appel à une culture scientifique globale et à une connaissance générale de la langue anglaise et du monde anglo-saxon, bien plus qu'à des concepts techniques.

Le thème de la publicité $\mathrm{a}$ également suscité un partage d'expériences pédagogiques. Marie-Hélène Doillon (INP, Université de Grenoble), utilise des publicités comme supports d'exposés, ce qui lui permet de varier, et de ranimer l'intérêt de son public d'élèves 
ingénieurs. Janet Atlan (IUT, Université de Nancy), fait composer des publicités orales à ses étudiants à partir d'un texte écrit, ce qui conduit à un travail fructueux sur la phonologie et l'intonation.

Hubert Greven a clos la séance en rappelant qu'en maîtrise de LEA, il demande même à ses étudiants de créer des films publicitaires, après avoir analysé avec eux des documents authentiques. Il nous a également proposé, comme objet de communication pour un prochain congrès, de " recréer "une publicité télévisée britannique où interviendrait le niveau phonologique, avec un problème stylistique de balancements de structures, d'échos et de rappels.

\section{NOTES}

1. Les publicités visionnées pendant la communication n'ont pu être reproduites pour des raisons pratiques, mais de plus amples informations sont disponibles auprès du pool des langues de l'Université Joseph Fourier. La liste des magazines dont sont tirées les publicités est donnée dans les références.

\section{AUTEUR}

\section{MARIE-HÉLÈNE FRIES-VERDEIL}

Université Joseph Fourier, Grenoble 1. Marie-Helene.Fries@ujf-grenoble.fr 